\title{
The soaring mechanic ventilator utilization under a universal health insurance in Taiwan
}

\author{
Shou-Hsia Cheng ${ }^{\mathrm{a}, *}$, I-Shiow Jan $^{\mathrm{b}}$, Pin-Chun Liu ${ }^{\mathrm{c}}$ \\ ${ }^{a}$ Institute of Health Policy \& Management and Center for Health Insurance Research, College of Public Health, \\ National Taiwan University, 17 Hsu-Chow Road, Room 618, Taipei 100, Taiwan \\ ${ }^{\mathrm{b}}$ Department of Laboratory Medicine, National Taiwan University Hospital, National Taiwan University College of Medicine, Taiwan \\ ${ }^{\mathrm{c}}$ Institute of Health Policy \& Management, College of Public Health, National Taiwan University, Taiwan
}

\begin{abstract}
Objectives: The use of mechanic ventilators (MVs) is increasing in many countries. Taiwan's Bureau of National Health Insurance (NHI) launched a new payment program in 2000 to encourage integrated care for mechanically ventilated patients and to reduce the heavy utilization of high-cost intensive care unit. This study examines the trend in MV usage in Taiwan.

Methods: This study used nationally representative NHI claim data from 1997 to 2004 to examine the MV usage. Total inpatient days and MV usage days were analyzed by piece-wise regression model. We also analyzed the major diagnoses related to MV dependence.

Results: While the total hospital inpatient days increased only $49.41 \%$, MV usage rose $181.75 \%$ over the 8 -year study period. The increase in number of MV patient-days in ICUs has not been curbed, besides there was an increase in respiratory care center/wards. Acute respiratory failure (ARF), pneumonia and diabetes mellitus were the leading diagnoses for mechanically ventilated patients.

Conclusions: The new NHI insurance payment program may have helped spark the increased trend in MV usage in Taiwan. The significant impact of insurance incentive on healthcare utilization is a critical issue for policymakers in developing healthcare programs.
\end{abstract}

(C) 2007 Elsevier Ireland Ltd. All rights reserved.

Keywords: Health insurance; Mechanic ventilator; Respiratory care; Taiwan

\section{Introduction}

The use of mechanical ventilators (MVs) has been on the rise in developed countries, particularly as their

\footnotetext{
* Corresponding author. Tel.: +8862 33228057 ; fax: +88622341 4634 .

E-mail address: shcheng@ntu.edu.tw (S.-H. Cheng).
}

populations age [1]. Many patients with respiratory failure require daily MV support via tracheotomy. Respiratory failure is often caused by chronic obstructive pulmonary disease (COPD), spinal cord injury, neuromuscular disease, acute lung injury, and primary central nerve system (CNS) disease [2,3].

Due to an increase in economic pressure to maximize utilization of resources in the 1990s in many 
countries, hospitals began transferring hemodynamically stable patients dependent on MV out of their ICUs into other wards or other facilities. One such facility is the regional ventilator weaning center, which has received a lot of research attention for several years in the US, UK, and other countries [3-16]. Many studies have reported that transferring MV-dependent patients from ICUs to regional ventilator weaning centers decreases costs $[8,17,18]$. Some have claimed that this change has brought about an increase in weaning rate and a decrease in mortality [19]. Most of these studies, however, are based on the experience of a single regional ventilator weaning center or respiratory care unit, which may not be an objective estimation. Furthermore, very limited reports have been revealed concerning the situation in Taiwan where the universal health insurance program has a great influence on healthcare delivery. This study investigates national trend in MV usage in Taiwan.

\subsection{The mechanic ventilator program in Taiwan}

Taiwan's National Health Insurance (NHI), a compulsory universal health insurance plan implemented in 1995, provides comprehensive healthcare coverage to all its residents [20]. It covers ambulatory and inpatient care, dental care, traditional Chinese medicine, medications, rehabilitation, and home health care. Previously, the use of MV was mostly confined to hospital ICUs. In 1998, the Bureau of NHI drafted a prospective payment program to encourage integrated care for mechanically ventilated patients. In order to reduce the heavy utilization of the high-cost ICUs, this program provided incentives for hospitals to increase the number of respiratory care wards. Then, in July 2000, the integrated prospective payment program for MV-dependent patients was implemented [21].

The NHI-integrated MV payment program covered four types of mechanic ventilator care: fee-for-service ICU care, per-day respiratory care center, per-monthbased respiratory care ward and per-month home healthcare services. The new payment program encouraged hospitals to expand or establish respiratory care centers for short stays and respiratory care wards for longer stays, and encouraged them to participate in its integrated payment program. A MV-dependent patient under the new program could stay 21 days in ICU and up to 42 days in a respiratory care center. Only large-scale teaching hospitals could apply to establish respiratory care centers, which provide comprehensive and intensive care to mechanically ventilated patients transferred from ICUs. According to the design of that new payment program, respiratory care centers would be for the care of MV patients with a greater likelihood of being weaned and respiratory care wards would be for the care of relatively stable MV-dependent patients with less likelihood of being weaned.

The new integrated MV payment program provides financial incentives to hospitals by raising the payment rates (to reduce the use of heavy-equipped high-cost ICUs). Hospitals with respiratory care centers participating in this new payment scheme are reimbursed at a much higher rate for each mechanically ventilated patient. Since 2001, the integrated payment scheme has been raised twice, once in 2002 and then in 2004. In 2004, the per-day payment was 9590 NTD within the program (1 USD $=33$ NTD) and it was 3900 NTD per day outside the program. The same was true for reimbursements to respiratory care wards. Participating hospitals with respiratory wards were reimbursed 92,230 NTD per month, while other hospitals were paid 727 NTD per day (about 21,810 NTD per month). The new MV payment program also reimburses hospitals for providing home healthcare services at a higher rate (27,610 NTD per month) than those not participating, which are paid on fee-for-service basis.

The main purpose of this study was to evaluate the effects of the integrated MV prospective payment program on trend in mechanical ventilator usage at the national level in Taiwan. The consequences of a nationwide insurance incentive program on MV usage may be of interest to other countries.

\section{Materials and methods}

The data for this study were collected from Taiwan's NHI Research Datasets which are provided by the Bureau of NHI and managed and distributed by the National Health Research Institutes in Taiwan. The NHI Research Datasets consist of two types of datasets: contracted healthcare provider profiles and insurance claim files. The contracted healthcare provider profiles offer information such as scrambled identification 
numbers, accreditation level and location of the hospital and also details of the contracted services provided by the hospital. The claim data files, made up of the claims submitted every month by the contracted healthcare providers for reimbursement purposes contain information on medical expenses, patient characteristics, diagnosis at the time of discharge and details of treatment orders.

In 2004, there were about 3 million hospital admissions for Taiwan's population of 23 million people. The specific datasets we used contained a random sample of $5 \%$ of the claims that had been submitted for all hospital admissions each year between 1997 and 2004 (currently available data sources). This national representative sample was obtained by using a systematic random sampling method each month, probability proportional to size of the inpatient claims, and then was compiled to form a sub-dataset of the entire claim data each year [22]. The dataset contained information on the patient's age, gender, admission and discharge dates, the primary diagnosis along with four secondary diagnoses, medical expenses as well as medication, examination and operation codes.

We calculated the trends for total inpatient days, ICU days, MV usage, and costs for these services. The inpatient claim dataset we used in this study included three types of the new integrated payment program for MV usage: MV usage in the ICU, respiratory care center and respiratory care wards. Home healthcare claims for MV-dependent patients were not reimbursed as hospi- talizations but as outpatient visits, and were, therefore, not included in this study.

While examining the trend for MV usage, we conducted a piece-wise regression using seasonal data to test for the impact of the integrated MV payment program implemented in July 2000 on the trend. We also collected data regarding diagnoses related to their MV dependence and compared them with reports from other countries.

Due to the characteristics of the sampling data from per-month claim files, we could not identify patients who stayed in the hospital across 2 months (about $3-7 \%$ of the admissions) or being transferred between hospitals, though the patient-days on MV were clearly recorded for reimbursement. Therefore, from this dataset we were unable to precisely calculate the average length-of-stay, weaning or survival rates for claimants admitted to ICUs, respiratory care centers or respiratory care wards.

\section{Results}

From 1997 to 2004, the total hospital inpatient days increased from $1,093,800$ days to $1,634,230$ days (a $49.41 \%$ increase), calculated from the $5 \%$ of the national hospitalization claims (Table 1). During the same period, we also observed the MV usage rose from 106,420 days in 1997 to 299,840 days in 2004. This represented a $181.75 \%$ increase in 8 years. The percentage

Table 1

Hospitalized patient-days and costs under the National Health Insurance in Taiwan, 1997-2004

\begin{tabular}{|c|c|c|c|c|c|c|c|c|c|}
\hline \multirow[t]{2}{*}{ Year } & \multicolumn{3}{|c|}{ Inpatient days ${ }^{\mathrm{a}}$} & \multicolumn{3}{|c|}{ ICU days ${ }^{\mathrm{a}}$} & \multicolumn{3}{|l|}{ Costs $^{b}$} \\
\hline & Total & $\mathrm{MV}^{\mathrm{c}}$ & $\%$ & Total & $\mathrm{MV}^{\mathrm{c}}$ & $\%$ & Total & $\mathrm{MV}^{\mathrm{c}}$ & $\%$ \\
\hline 1997 & 1093.80 & 106.42 & 9.73 & 50.62 & 38.53 & 76.12 & 4.06 & 0.94 & 23.15 \\
\hline 1998 & 1066.24 & 110.50 & 10.36 & 58.68 & 45.81 & 78.07 & 4.99 & 1.12 & 22.44 \\
\hline 1999 & 1162.49 & 138.86 & 11.95 & 64.82 & 52.38 & 80.81 & 5.53 & 1.31 & 23.69 \\
\hline 2000 & 1148.76 & 149.74 & 13.03 & 63.95 & 51.56 & 80.63 & 5.70 & 1.41 & 24.74 \\
\hline 2001 & 1291.44 & 187.98 & 14.56 & 72.32 & 58.82 & 81.33 & 6.25 & 1.76 & 28.16 \\
\hline 2002 & 1386.00 & 216.13 & 15.59 & 78.48 & 64.29 & 81.92 & 6.94 & 2.05 & 29.54 \\
\hline 2003 & 1447.27 & 238.88 & 16.51 & 82.22 & 66.69 & 81.11 & 7.09 & 2.31 & 32.58 \\
\hline 2004 & 1634.23 & 299.84 & 18.35 & 91.84 & 74.89 & 81.54 & 8.43 & 2.69 & 31.91 \\
\hline Change rate ${ }^{\mathrm{d}} \%$ & 49.41 & 181.75 & & 81.43 & 94.37 & & 107.64 & 186.17 & \\
\hline
\end{tabular}

\footnotetext{
a Thousand days.

${ }^{b}$ In billion New Taiwan Dollars.

c Mechanic ventilator usage.

${ }^{\mathrm{d}}$ Change rate between the years 1997 and 2004.
} 


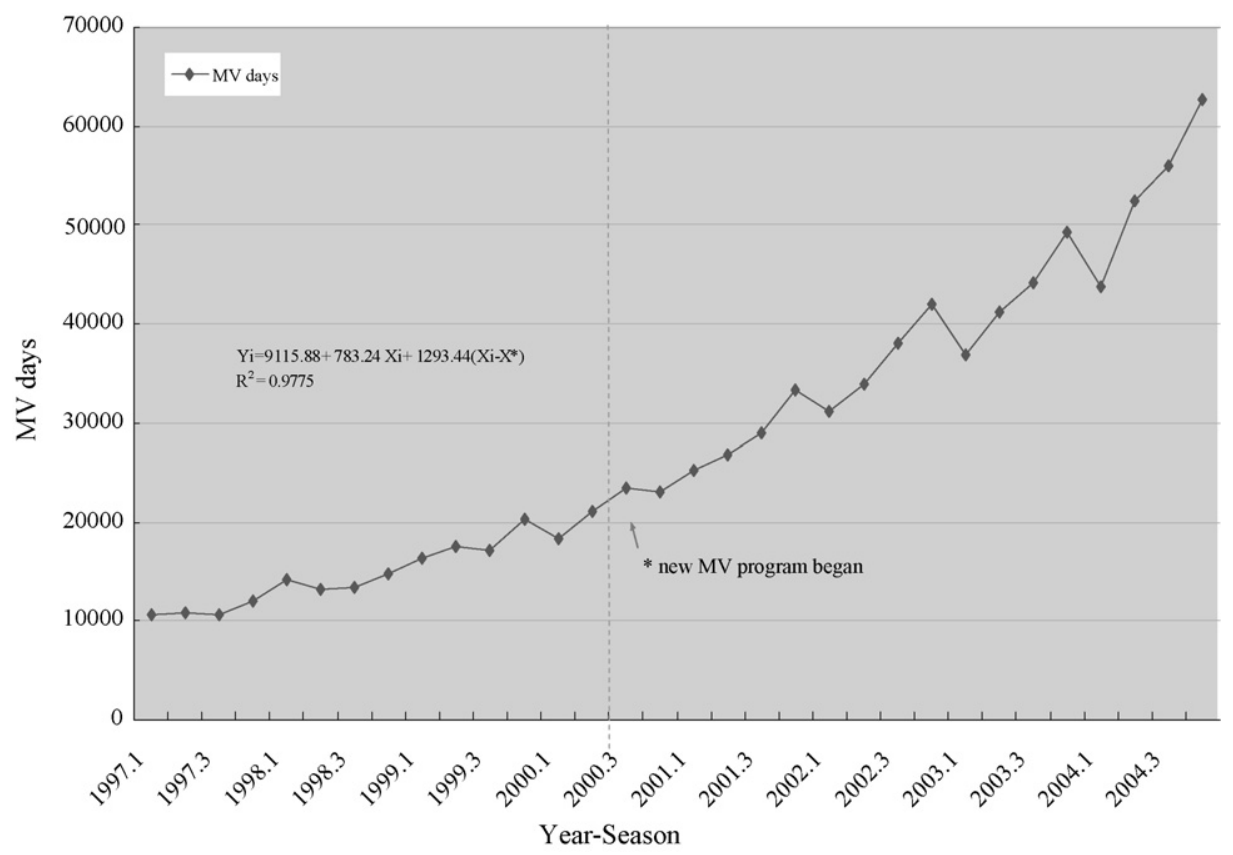

Fig. 1. The trend of mechanic ventilator (MV) patient-days in Taiwan, 1997-2004.

of the MV patient-days to total patient-days increased from 9.73 to $18.35 \%$.

Results from the piece-wise regression model indicated that with the introduction of the NHI-integrated MV payment program in July 2000 there was a significant increase in MV usage (Fig. 1). Before the introduction of the new MV payment program, the average MV patient-days increased 783.24 days per season, while after the new program the average MV patient-days increased $2076.68(783.24+1293.44)$ days per season.

Since the integrated MV payment program was introduced mainly to reduce the cost of treating mechanically ventilated patients in ICUs, we compared ICU patient-days with MV usage days in ICUs (Table 1). The ICU patient-days increased from 50,620 days in 1997 to 91,840 days in 2004, an $81.43 \%$ increase. Meanwhile, over the same period, MV days in the ICU setting increased from 38,530 to 74,890 days, a $94.37 \%$ increase. The ratio of ICU MV patient-days to total ICU days rose from $76.12 \%$ in 1997 to $80.63 \%$ in 2000. After that, figures remained stable at around $81 \%$ from 2001 to 2004.

We also found a significant increase in the MV usage in settings other than ICUs between 1997 and 2004.
In 1997, 36.2\% (38.53/106.42) of the MV patient-days were spent in ICUs, while in 2004 that figure dropped to $25.0 \%$ (74.89/299.84). However, we noticed that there was a large 193,420-day increase in total MV-days (106,420 days vs. 299,840 days) but only a $36,360-$ day increase in ICU MV-days (38,530 days vs. 74,890 days) during the 8 -year study period. These findings showed that MV usage increased most in non-ICU settings such as respiratory care centers and respiratory care wards. This situation resulted in increased MV expenses.

The final part of Table 1 shows the costs of total inpatient services as well as the costs incurred by MV-dependent patients. From 1997 to 2004, the total hospital inpatient costs increased from 4.06 billion NT dollars to 8.43 billion NT dollars, a $107.64 \%$ increase. While MV-dependent inpatient costs increased from 0.94 billion NT dollars to 2.69 billion NT dollars, a $186.17 \%$ increase. The percentage of the costs spent by MV-dependent patients to that of total hospitalized patients rose from $23.15 \%$ in 1997 to $31.91 \%$ in 2004, mainly in non-ICU settings. All the figures in Table 1 indicate a noticeable increase of the utilization of mechanic ventilator in Taiwan. 


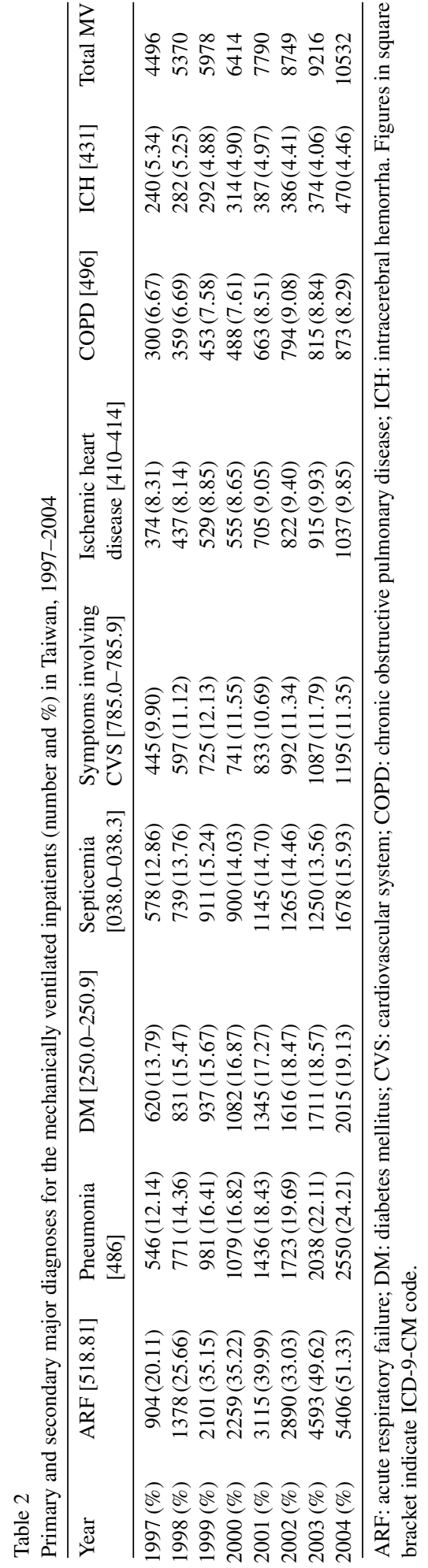

Table 2 shows the major diagnoses, one primary diagnosis and up to four secondary diagnoses, for hospitalized patients using MV. In addition to finding that the number of patients using MV increased from 4496 in 1997 to 10,532 in 2004, we found the eight most prevalent diagnoses related to the use of MV were acute respiratory failure (ARF), pneumonia, diabetes mellitus (DM), septicemia, symptoms involving cardiovascular system, ischemic heart disease (IHD), COPD and intracerebral hemorrhage (ICH). In 2004, $51.33 \%$ of all MV patients claimed ARF as their major and secondary diagnosis, followed by pneumonia $(24.21 \%)$ and DM (19.13\%).

\section{Discussion}

This study investigated the impact of the NHIintegrated MV payment program implemented in July 2000 on trend in MV usage. We found that between 1997 and 2004 while the total hospital inpatient days increased only about 50\%, MV patient-days increased dramatically by $180 \%$. We found the largest MV patient-day increases to occur in setting outside the ICU. Therefore, the dramatic increase in the MV usage was significantly associated with the introduction of that integrated MV payment program, which encouraged the expansion of hospital-affiliated respiratory care centers and respiratory care wards nationwide.

Has the integrated MV payment program reduced the use of high-cost ICU services by mechanically ventilated patients? The percentage of ICU MV patientdays out of total ICU days rose from 76.12\% in 1997 to $80.63 \%$ in 2000 , after which the figures stabilized to around $81 \%$ from 2001 to 2004 . Though it might have curbed the increases that were occurring in ICU settings at the time of implementation, there was no evidence that it had decreased the use of mechanic ventilators in the ICU setting. One NHI document also reported a shortened average MV-days per patient stay in ICU settings [23]. In addition, doctors also revealed that the arrangement of ICU beds became more flexible after the expansion of respiratory care centers thus might save more critical patients who needed ICU care.

Since the dramatic increase of MV occurred in non-ICU settings, was MV usage appropriate? Due to limited evidence we can only discuss this important issue preliminarily. We examined the diagnoses of 
MV-dependent patients first. The most prevalent primary or secondary major diagnosis for the mechanically ventilated patients in Taiwan was ARF, which is different from that reported in the US or other countries, where COPD is the most frequently reported $[3,4,10,13]$. The Bureau of NHI stipulates that only patients with certain respiratory failure diagnoses are eligible to claim reimbursement for MV usage. Therefore, any patient who needed MV would be assigned a respiratory failure related diagnosis as one of the diagnosis codes in the medical claims to better fit the NHI rules. Physicians in Taiwan revealed that ARF tended to be the easiest and a safe code for patients who needed MV (which complied with the requirement of the NHI), and thus might explain why our MV-dependent patients had a higher prevalence of ARF as primary or secondary diagnoses. Apart from this difference, the diagnoses were similar to those reported by other studies.

The other important issue is the outcome of the respiratory care centers and wards. According to a summary report on the first 2 years (July 2000 to June 2002) of the integrated MV payment program from the Bureau of NHI [23], the weaning rate of the patients in respiratory care centers (in large-scale hospitals) was about $30.8 \%$ and that was about $5 \%$ in respiratory care wards (mostly in small-scale hospitals). The overall mortality rate of these patients was very high, around $47 \%$. Local studies of respiratory care centers in several large-scale medical centers have reported higher weaning rates, range from 35 to $70 \%$, and lower mortality rates from 10.3 to $16.3 \%$ [24-27], compared with the above-mentioned summary report. These reported weaning rates in respiratory care centers taking patients transferred from ICUs in large-scale hospitals were close to those respiratory care unit or ventilator weaning centers reported in the US and the UK, ranging from 25 to $82 \%$ [18]. However, the extremely low weaning rate of $5 \%$ and very high mortality rate of $47 \%$ in respiratory care wards seemed unusual in Taiwan.

The majority of respiratory wards are set-up inside small-scale hospitals. The fact is that many of the small-scale hospitals are less competitive in acute care services and with low occupancy rates [28], and gradually transferred into a chronic care oriented facility. The NHI MV payment program provides them a chance to survive. Some are worried that the very low weaning rate might be due to the NHI financial incentive for the hospitals to keep the patients in the respiratory care wards without trying to have the ventilated patients weaned actively. Another explanation to the situation is that the payment to the hospitals for home health care is much less than for the respiratory care wards inside the hospital. This issue is out of the scope of this study; however it is important to the healthcare system in Taiwan.

In conclusion, our study found that the new integrated payment program for MV-dependent patients caused a dramatic increase in MV usage in Taiwan. While the program did not reduce the ventilated patient-days in ICU settings, the appropriateness of the increased mechanic ventilator usage, especially in many respiratory care wards, deserves further investigation. The significant impact of insurance incentive on healthcare provision and utilization is a critical issue for policy makers in developing healthcare programs.

\section{Acknowledgements}

The study was supported by grants from the National Health Research Institutes (NHRI-EX94-9310PI) and the National Science Council (NSC95-2416-H-002015) in Taiwan. Data of the study came from NHI Research Datasets which are provided by the Bureau of NHI and managed and distributed by the National Health Research Institutes in Taiwan. The authors are grateful to the Aiming for the Top University and Elite Research Center Development Plan (96HP0021), Mr. James Steed for his English editing of this manuscript, and Ms. Chi-Chen Chen for her assistance in preparation of this manuscript.

\section{References}

[1] El Solh AA, Ramadan FH. Overview of respiratory failure in older adults. Journal of Intensive Care Medicine 2006; 21:345-51.

[2] Votto J, Brancifort JM, Scalise PJ, Wollschlager CM, Zuwallack RL. COPD and other diseases in chronically ventilated patients in a prolonged respiratory care unit: a retrospective 20 -year survival study. Chest 1998;113:86-90.

[3] Bagley PH, Cooney E. A community-based regional ventilator weaning unit: development and outcomes. Chest 1997;111:1024-9.

[4] Gracey DR, Viggiano RW, Naessens JM, Hubmayr RD, Silverstem MD, Koenig GE. Outcomes of patients admitted to 
a chronic ventilator-dependent unit in an acute-care hospital. Mayo Clinic Proceedings 1992;67:131-6.

[5] Scheinhorn DJ, Artinian BM, Catlin JL. Weaning from prolonged mechanical ventilation. The experience at a regional weaning center. Chest 1994;105:534-9.

[6] Scalise PJ, Gerardi DA, Wollschlager CM, Votto JJ. A regional weaning center for patients requiring mechanical ventilation: an 18-month experience. Connecticut Medicine 1997;61:387-9.

[7] Dasgupta A, Rice R, Mascha E, Litaker D, Stoller JK. Four-year experience with a unit for long-term ventilation (respiratory special care unit) at the Cleveland Clinic Foundation. Chest 1999;116:447-55.

[8] Gracey DR, Hardy DC, Koenig GE. The chronic ventilatordependent unit: a lower-cost alternative to intensive care. Mayo Clinic Proceedings 2000;75:445-9.

[9] Modawal A, Candadai NP, Mandell KM, Moore ES, Hornung RW, Ho ML. Weaning success among ventilator-dependent patients in a rehabilitation facility. Archives of Physical Medicine Rehabilitation 2002;83:154-7.

[10] Schonhofer B, Euteneuer S, Nava S, Suchi S, Kohler D. Survival of mechanically ventilated patients admitted to a specialised weaning centre. Intensive Care Medicine 2002;28:908-16.

[11] Criner GJ. Long-term ventilation introduction and perspectives. Respiratory Care Clinics of North America 2002;8:345-53.

[12] Robson V, Poynter J, Lawler PG, Baudouin SV. The need for a regional weaning centre, a one-year survey of intensive care weaning delay in the Northern Region of England. Anaesthesia 2003;58:161-5.

[13] Ceriana P, Delmastro M, Rampulla C, Nava S. Demographics and clinical outcomes of patients admitted to a respiratory intensive care unit located in a rehabilitation center. Respiratory Care 2003;48:670-6.

[14] Lindsay ME, Bijwadia JS, Schauer WW, Rozich JD. Shifting care of chronic ventilator-dependent patients from the intensive care unit to the nursing home. Joint Commission Journal on Quality and Safety 2004;30:257-65.

[15] Pilcher DV, Bailey MJ, Treacher DF, Hamid S, Williams AJ, Davidson AC. Outcomes, cost and long term survival of patients referred to a regional weaning centre. Thorax 2005;60:187-92.

[16] Cohen J, Starobin D, Papirov G, Shapiro M, Grozovsky E, Kramer MR. Initial experience with a mechanical ventilation weaning unit. The Israel Medical Association Journal 2005;7:166-8.
[17] Seneff MG, Wagner D, Thompson D, Honeycutt C, Silver MR. The impact of long-term acute-care facilities on the outcome and cost of care for patients undergoing prolonged mechanical ventilation. Critical Care Medicine 2000;28:342-50.

[18] Nevins ML, Epstein SK. Weaning from prolonged mechanical ventilation. Clinics in Chest Medicine 2001;22:1333.

[19] Scheinhorn DJ, Chao DC, Stearn-Hassenpflug M, LaBree LD, Heltsley DJ. Post-ICU mechanical ventilation: treatment of 1,123 patients at a regional weaning center. Chest 1997;111:1654-9.

[20] Cheng TM. Taiwan's new national health insurance program: genesis and experience so far. Health Affairs 2003;22:6176.

[21] Bureau of National Health Insurance. NHI announcement, retrieved at February 2, 2006 from http://www.nhi.gov.tw/ information/law_detail.asp?Bulletin_ID=74\&menu=1\&menu _id=9\&IsPrint $=1 . \% 20$ Accessed $\% 20$ Feb. 2.2006 [Chinese].

[22] National Health Research Institute. Overview of random samples of NHI beneficiaries, retrieved at September 29, 2007 from http://www.nhri. org.tw/nhird/date_cohort.htm [Chinese].

[23] Bureau of National Health Insurance. NHI announcement, retrieved at February 2, 2006 from http://www.nhi.gov.tw/intro/statistic/work9112-3.ppt [Chinese].

[24] Lee JD, Chen PP, Lin BH. Long term mechanical ventilation. Journal of Respiratory Therapy 2003;2:21-6 [Chinese].

[25] Kung SC, Cheng KC, Shih MJ, Hou CC. Evaluation of the effects of the ventilator weaning protocol in the respiratory care center. Journal of Emergency and Critical Care Medicine 2004;15:93-102 [Chinese].

[26] Huang MH, Chang HY, Yen LC, Lin WC, Chu YC, Chen CZ. Outcomes of patients after discharge from the respiratory care center. Thoracic Medicine 2006;21:16-24.

[27] Chen CC, Sung MY, Cheng KC, Shieh JM. The relationship between body mass index and prognosis in a respiratory care center. Journal of Emergency and Critical Care Medicine 2005;16:9-17 [Chinese].

[28] Occupancy rate of hospital beds by accredited hospitals in 2006, retrieved at September 29, 2007 from http://www.doh.gov.tw/statistic/ [Chinese]. 